\title{
On Musical Notations
}

Andrei Kim

Deviations from traditional notations seem counterintuitive-- as the norm works well. The reason why such a system holds an important place is because centuries of work, over many cultures and languages, did go into establishing it, a grammar was both induced and deduced upon agreement in collaboration with different professions: composers, theorists, the musicians themselves, had to come up with a method verified by consensus. Once such a system has reached a point of stability, further developments seem regressive: a perfect world would be one where the entirety of those involved agreeing upon a halt of development, upon the absence of any further trials and errors onwards. Such a thing never seems to happen in any sort of system: "graphic" notations were developed onwards from the traditional system-- defying and reconstructing the rules that were held in place by consensus, perhaps as genuine development, possibly as regression, or even the destruction of that which was in place.

The origins of the traditional staff is often attributed to Guido of Arezzo, around 1030, proposing "ruled staff" for "pitch content". ${ }^{1}$ Scholars, having found developments and prototypes before Guido, are more hesitant to define Guido as the sole inventor and creator-- and deemed him only having "perfected staff notation" by improving "an imperfect system already in use". 2 What came before was pragmatic "prickings" as systematic lines for texts to be written upon-developed out of necessity, "fundamental to the existence of... the staff". ${ }^{3}$ Subtle variations onwards "adapted existing ruling lines" for texts, leading to "greater proportion and symmetry", increasing space for musical notations in between the texts, and near the thirteenth century after experimentation with colouring the staff etc., returned back to "evenly spaced pricking and ruling pattern", but having been finalized for musical notations. ${ }^{4}$ Guido, as opposed to such generalizations for ease of reference, was responsible for having "popularized these use of multiple rulings in common music books... rather than an [inventor] of the staff... [a] historical [contributor] to its development". ${ }^{5}$

Is there, ever, such a proper origin to a development to an idea? Can one pinpoint, with proper evidence, the single historical point whereupon all consequent developments occurred out of? Had Guido been the sole origin, further explanations in hindsight would be, to say the least, easier-- such ease of reference to a person would save scholars a lot of time, forgetting that Guido himself was necessitated to work under contingencies of a specific time and place to conjure up his developments into popularization. Furthermore, when is such a development finished? When does it become fully "traditional" in essence? The ending of such a development is equally as difficult to develop a consensus upon-- perhaps all there is, in essence, is merely such a consensus: after Guido, the "innovation of hundreds if not thousands of nameless

\footnotetext{
${ }^{1}$ Haines, John. "The Origins of the Musical Staff." The Musical Quarterly, Fall-Winter 2008, 91, no. 3-4

(May 4, 2009): 327-78. doi:10.1093/musqt//gdp002. 327.

${ }^{2}$ Ibid. 328.

${ }^{3}$ Ibid. 337.

${ }^{4}$ Ibid. 367.

${ }^{5}$ Ibid. 344.
} 
monks... lowly workers in the service of their order, religion, and craft". ${ }^{6}$ The impossibility of a perfect single source, simultaneously with a pragmatic necessity for its ease of reference, is a historical paradox and a problem that contrasts its contingencies along with its necessities in hindsight. The finalities are as equally diluted as the origins-- no one can say for sure the point when traditional staff becomes "traditional" in its entirety.

The traditional staff is supposed to be read left to right-- once one horizontal line is done the reader moves to the line below, much like traditional writing. The form, or the space that pictorial signs are put onto, is a series of five horizontal lines, that constitutes four spaces in-between the lines. These formal sets are repeated as much as they need to serve the music. At the very left is the information necessary to put the whole of the reading into context-whether it is in the treble or the bass clef, the tonal key marked by its corresponding placements of the groupings of flats and sharps in such a key, along with the time signature-- all before the actual notation of the music itself starts. The form that the content necessarily adheres to-- the placement of the register, the mode of pitch, the numerical division of the value of time-- is transcribed within the same realm: the contextual agreement of the music happens on the same plane as the transcription of the music, which happens accordingly to such agreements.

Horizontally, time flows from left to right. This is not at all that different from reading language: a moment in time is the focus of the eye onto the score-- hindsight would be tracing the focus backwards (upwards and to the left) to revisit that which has passed, whilst a foresight would be tracing the focus forwards to visit that which is yet to come. All moments in time in the music is laid out at once in notation, the reader having the free will to move forwards and backwards in time, ideally having mastered the piece time will "flow" as the player or the reader reads through it, without slippages or interruptions. The speed and direction of such horizontality, then, is simultaneous in nature, and determined by the reader.

Within the time frame are absences and presences of sound values placed throughout. A presence of a note as an entity is a circle, or an oval, onto either the lines or the spaces in-between: stems growing out of it and the manners thereof indicates the time-value that each notes exist for. The shorter the note value, the more complicated the stem seems to be: a "thirty-second" note, lasting for $1 / 8$ of a beat, has three additional stems rooting out from the original, one for each further division of its own time halved, all the way from the eighth note. Neither half notes or full notes are coloured in-- a whole note doesn't even have a stem, the simplicity of the whole is the basis of these timed entities, that which is a near perfect circle, denoting, strangely, not an undivided "whole" beat, but 4 beats. Complexity is added not as addition onto the whole, but a division into the whole, as the pictorial schemes become further and more complicated from the original oval. The rests, or the denotations of the absence of a sound seems to be a bit less schematic and more random visually, but follows the same temporal divisional schemes that the notes as presences are subject to.

The temporal centre as a number, the whole, strangely, is the number 4 , and not number 1 -- which is a "quarter" note. 1 is that which 4 grows out of, via self-duplication, from

${ }^{6}$ Ibid. 369. 
quarter to a half to a whole. 1 is also that which is divided upon in halves, from quarter, to eighth to the sixteenth-- it is debatable, then, which number holds music together: is it that which is denoted as "whole", 4 in its completion, as a near perfect circle, or the quarter note as the foremost "being" of 1 , that which makes, say, both 4 and $1 / 4$ possible in the first place? 4 seems to have more of a semblance of such wholeness, because a self-duplication upwards from 1 , to 2 , to 4 , must have a semblance of an end before it goes on ad infinitum. Doing it twice, then, might have seemed like an apt sense of completion, before "double whole" or "quadruple whole" notes, which could be unnecessary. 4 could be more a sense of finality as denotation of "the whole", whilst 1 is as the real centre, that which makes all the other numbers in music possible, even the numbers that come in-between standard divisions, "dotted half" and "dotted quarter" notes and rests, etc.

Vertically, then, pitch is indicative and final: there is less of a choice. One can't subvert pitch values as they could speed up and slow down the horizontal process of reading. The reader has the choice over the speed at which they read, but they can't change the innate note values to rearrange that which is already there-- it would be a different piece of music. Pitch divisions, having been standardized in ratio by equal temperament, with 12 notes in each octave, is the basis for such verticality. Sensibly, the higher one goes up on the staff, the higher the pitch value, and vice versa. Each "movement", either upwards or downwards, from the space between to the line or vice versa, is indicative of a single semitone, one-twelfth of an octave. Often ledger lines as extensions are used above or below the initial five lines to indicate that which is higher or lower than that which is within the allotted five lines. The presences and absences of such note values are placed within time, in rhythmical succession, and often in harmonic conjunction or disjunction with one another to make pitch as verticality possible, only amidst time as horizontality. On top of such formalities are commonly used accessories, which are also in consensus: grace-notes, glissandos, accented notes, etc., to serve the manner of pitch values, timbre of the notes, stranger intonations-- whilst seeming more contingent, such stylistic choices might equally make up the whole of musical notations, and consequently music, as more of an inscription of either the composer or the player, with the demands of interpretations being usually slightly more loose in such cases.

The verticality as definitively measured content of pitch values is necessitated to be in collaboration consistently with horizontality as freedom of spatial time (which is essentially simultaneous as a whole) for the reading of traditional staff to work. There is a dependency, then, of self-definition on interpretation, of pitch on time, of verticality on horizontality, etc. Essentially, musical notation isn't possible without the interpretation of the reader. The manner of notation, inevitably, necessitates the different subjective manners of interpretation by the reader, and quite possibly the disjunction and conjunction of the opinions of interpretations for it to work properly-- the pitch values wouldn't mean anything if it isn't displayed across horizontally, as much as such display of time wouldn't mean anything if it isn't for the pitch values and the absences thereof that are indicated amidst it. Strangely, a piece of music as musical notation seems to defy being a pure object-- as what is necessary was a self-definition of the composer of the music as the subject, simultaneously with the interpretation of the player or the reader as the reflection of the subject. Inevitably, it is that which is a method of 
communication between the two subjects, mediated by an (what seems like) necessary form and content, possibly at first glance without a semblance of historicity, due to its seeming cogency and self-evidence. Learning of such history, then, reveals the strangeness of what seems like perfection (eg. "4" as "whole"), the dilution of that which seemed stable, just, and ultimately safe. There is no perfect system, as much as there is no such thing as a perfect musical piece: the traditional staff, then, is only "traditional" within the context of a certain historical time and space-- that which is quite Western and inevitably eurocentric, only having the hindsight can theorists define it as traditional, against that which wasn't and that which has become.

"Graphic" notations, then, stem from such developments, it is what traditional notation inevitably has become over the course of time. What was such form and content, having the seemingness of self-evidence and stability, could've been bored of itself, felt the need to play with its own rules, to the point where a lot of itself was disregarded in search of something new. Composers such as Crumb, Xenakis, and Cage all play with the mannerisms of notations deviating from the aforementioned description of the "traditional" "staff", such stylistic mannerisms, possibly, becoming akin to or borrowing from visual arts, as if the process of the different manners of visualization are less the mark of a composer, but equally that of a visual artist. The musical notation has less of a value in that it accurately and properly matches the auditory experience, and gains more of a value in its originality in the mannerisms of visual depiction, the amount of deviation from the norm, and so on. Much like the development of traditional notation out of itself, a particular origin and a consequent genealogy are probably more difficult to trace backwards, hence such an attempt not being an apt focus-- rather the particularities of graphic notation itself, the examination of the particularities of different pieces that utilize graphic notations, what it means and whether it matters at all.

Crumbs' Spiral Galaxy, a piece within Makrokosmos, utilizes such graphic notation-- the notation is a spiral into itself, starting at the outer edges and ending at the innermost. Instead of linear lines, the whole of the notation is consistently curved, having no separation from one line to another. Consequently, both horizontality and verticality within the context of the notation still exist. Time as traditionally notated still exists-- the piece is still read left to right, albeit consistently in rotation. Pitch as traditionally notated also still exists, and there is no major variation from the traditional staff. What has changed, however, is the space around the traditional staff, having been bent, an allowance for continuity, instead of discrete separation due to the lack of space. The rigidity of the space around the former traditional staff is gone, and what is left is a fluid space where the piece circles around into itself. Spiral Galaxy, auditorily, does feel like a quiet descent, or an ascent into itself, being brief and tame-- a continual progression without much happening essentially, and visually, the manner in which Crumb decides to subvert the notation does mirror what is happening in the music. The notation gains its value, not for its accuracy, not for its ease of access, but having the manner of notation reflect the way the piece feels.

Xenakis' Pithoprakta is of incredible mathematical complexity to determine the piece's note placement and direction, which is all better left out, the focus being purely on the graphic 
notation itself. Unlike Spiral Galaxy, there is no traditional notation left at all, but merely purely pictorial forms to indicate pitch and timbre through time. Furthermore, unlike Spiral Galaxy, there is a horizontal linear progression of time left to right-- and placed within the score are dots, diagonal lines, and scribbles: the pitch and timbre of which are mimicked by live instruments. What seems like a mash of sine waves sounds exactly like it looks: pitch oscillates upwards and downwards, as glissando converges and diverges with each other. Scattered dots are percussion hits: often there are cascades of them all at once, leaving the listener to wonder how the players keep in sync and know what to play when. Short horizontal dashes are bursts of sound from the instruments with a consistent pitch, interlaced with diagonal lines and often what seems like mere scribbles-- which sounds exactly as it looks like. Strangely enough, then, the main form of time as horizontality, along with pitch as verticality remains the same. What has changed is the presence of notes, which is a lot more pictorial than the traditional staff notation. Lines, dashes, and scribbles replace notes and its absences-- there are no horizontal divisions, nor the spaces in-between: pitch and timbre, in turn, become a realm of play auditorily, as much as the graphic notations visually.

Cage's Water Walk might be the most free form of these graphic notations-- it disregards all former traditional staff requirements: the metre division is purely temporal, in intervals of five seconds. There are no notes, no pictorial or traditional placements of them, but merely often vague instructions for the player to follow: "Slam lid", "Vase + Roses in tub", and "Fill pitcher + put near whistle". Time is still read left to right, but instead of divisions thereof, time becomes absolute: the minutes and seconds that are standardized are what is used to keep track of time throughout the piece. There are pictorial markings, but they are literally pictorial: pianos, bottles, and what seems like a fish accompanies the captions as instructions as they are. No longer are visual markings formal, in reference to pitch and timbre, or even really regards to the music in general at all, they seem silly, humorous, and often seems to be making fun of itself-- in the process testing the limits of what graphic notation could become. Cage plays with the boundaries of not ideas within music, but music itself: what constitutes, conceptually as music-consequently such ideas seeping into how he visually notates his pieces. Conceptually, it becomes self-reflexive: more questions are raised about the nature of "musical" "notations" and the boundaries thereof, than any antecedent theories-- pitch, timbre, etc. Fundamentally, via self-reflexivity, Cage seems to be saying through the piece, both musically and visually, that there are no inherent boundaries of that which constitutes what is and isn't music, merely consensus and agreements that determine such. Value, then, is given to the piece by the freedom of experimentation-- the manners in which self-reflexivity is allowed: often just by ignorance of that which came before. ${ }^{7}$

\footnotetext{
${ }^{7}$ Cage's 4'33" uses the past by absolutely disregarding all of it-- complete silence, the absence of all music that came before, and regarding it as music. That which is music becomes more about that which had been music, and the absence thereof, being integrated and pushing the boundaries of what can and cannot be defined as music. The past as inspiration, then, is music, whilst he negates his inspiration completely, integrating what was left without (complete silence) within the boundaries of music (presence of sounds). Music itself becomes pliable, and probably strays further from what it traditionally deemed itself to be after Cage, as he plays with the conceptual boundaries of what music is more than what is important musically and theoretically, which is not at all that different to what was happening in modern art at the time anyways.
} 
Do the deviations in graphic notations make a lot of difference musically? Does it change the trajectory of music as a whole? Having been an accompaniment, the notation-- as it turns from traditional to graphic-- become more a standalone piece. The visual no longer merely serves the music, but is also able to be examined on its own for its own right-- the stylizations and mannerisms of visual graphic notations are of equal importance as that of the music. The trajectory of musical development is now able to be influenced by the newfound trajectory of the development of graphic notations, and vice versa-- whereas in the past the realities of the visual accompaniment arose out of a pragmatic necessity for the music, the development of graphic notations, then, is almost like a creation of a new world that works in conjunction and disjunction along with music, an added complexity as feedback loop, a new dimension that has an influence onto and within each other. Some can call it enhancement, others can call it unnecessary complexity-- what cannot be ignored is that such development has been made in the twentieth and twenty-first centuries.

Is it necessary? Does it matter? Where does music and its notations go from here? Perhaps the development of graphic notations, is akin to the beginnings of the development of the traditional staff-- its origins, and its finalities of development, would be equally as diluted in hindsight in the future. A "Guido" of graphic notations might be Crumb, Xenakis, Cage, or any other attempts: randomized graphic notation could precede the music, randomized music can produce, mechanically, its corresponding graphic notations, and so on. The realm of historical play of both a necessary semblance of an origin along with the proper impossibility thereof doesn't disappear within the realm of graphic notations, the developments further hereon are possibly as unforeseen as Crumb would have been in Guido's time. Does it make a difference? Where from here? Such developments might be the present moment itself, needing to work the past into the future. 


\section{Works Cited}

Haines, John. "The Origins of the Musical Staff." The Musical Quarterly, Fall-Winter 2008, 91, no. 3-4 (May 4, 2009): 327-78. doi:10.1093/musqt//gdp002. 